\title{
Consequences of the Technology Survey and Gap Analysis on the EU DEMO R\&D Programme in Tritium, Matter Injection and Vacuum
}

\author{
Chr. Day $^{\mathrm{a}}$, B. Butler ${ }^{\mathrm{b}}$, T. Giegerich ${ }^{\mathrm{a}}$, P.T. Lang ${ }^{\mathrm{c}}$, R. Lawless ${ }^{\mathrm{b}}$, and B. Meszaros ${ }^{\mathrm{d}}$ \\ ${ }^{a}$ Karlsruhe Institute of Technology (KIT), Karlsruhe, Germany \\ ${ }^{b}$ Culham Science Centre (CCFE), Abingdon, UK \\ ${ }^{c}$ Max-Planck-Institute of Plasma Physics (IPP), Garching, Germany \\ ${ }^{d}$ EUROfusion Consortium, Programme Management Unit, Garching, Germany
}

In the framework of the EUROfusion Programme, EU is preparing the conceptual design of the inner fuel cycle of a pulsed tokamak DEMO. This paper illustrates a quantified process to shape a R\&D programme that exploits as much as possible previous R\&D. In an initial step, the high-level requirements are collected and a novel DEMO inner fuel cycle architecture with its three sub-systems vacuum pumping, matter injection (fuelling and injection of plasma enhancement gases) and tritium systems (tritium plant and breeder coolant purification) is delineated, driven by the DEMO key challenge to reduce tritium inventory. Then, a technology survey is carried out to review potential existing solutions for the required process functions and to assess their maturity and risks. Finally, a decision-making scheme is applied to select the most promising candidates. ITER technology is exploited where possible. As a primary result, a fuel cycle architecture is suggested with an advanced tritium plant that avoids full isotope separation in the main loop and with a Direct Internal Recycling path in the vacuum systems to shorten cycle times. For core fuelling, classical inboard pellet injection technology is selected, in principle similar to that proposed for ITER but aiming for higher launch speeds to achieve deep fuelling of the DEMO plasma. Based on these findings, a tailored R\&D programme is shaped that tackles the key questions until 2020.

Keywords: fuel cycle; DEMO; vacuum; tritium; pellet injection

\section{Introduction}

EU is currently working on a pre-conceptual design of a pulsed demonstration fusion power plant (DEMO) to implement the "Roadmap to the realisation of fusion energy" [1], which is currently being updated to reflect the results of the review of the ITER overall schedule and associated resources, and other recent developments in the worldwide fusion programme. This European roadmap sets out the strategy to achieve the goal of generating fusion electricity by 2050. DEMO adds three roles that cannot be addressed in ITER: first, it will demonstrate self-sufficiency by breeding its own tritium fuel in situ; second, it will utilise materials that are able to withstand radiation doses over periods of several years; third, DEMO will convert fusion energy into electricity and feed it into the grid. The latter aspect requires a potentially high availability and promising commercial attractiveness of DEMO in view of future fusion power plants. This marks another difference between DEMO and ITER: ITER is an experimental device which has been designed in such a way that a wide operational window is possible which allows for parametric variation of physics and technology parameters during experimental campaigns, whereas the design of DEMO routine operation will be focused on one single operational point and the operational window is only defined by an uncertainty and control stability frame around this operational point. However, this point will have to be identified to a significant extent by experiments. It is obvious that this fundamental difference asks for a new development approach beyond that taken for ITER.
The roadmap elaborates eight strategic missions to tackle the major challenges. The inner fuel cycle (together with its three sub-systems tritium processing (except tritium generation and extraction from the blankets), matter injection, and vacuum) is deeply involved in two of them. Mission 4 (Tritium selfsufficiency) addresses the tritium plant systems: DEMO will substantially benefit from the experience gained in the operation of the ITER fuel cycle system [2, 3], but a development in the field of removal and processing of tritium from candidate breeder blanket systems in large scale will be needed to reduce the processing time, thereby improving system availability. Mission 6 (Integrated DEMO design and system development [4, 5]) adds the fuelling and pumping systems. The need for a self-sufficient tritium fuel cycle and hour long scale plasma pulse durations requires systems with performance characteristics beyond those considered for ITER. The proper analysis of DEMO requirements, system modelling and design integration of the various systems that form the DEMO plant is key to the success of Mission 6.

Some assumptions have been made about the operation of the DEMO inner fuel cycle in order to undertake this work:

- DEMO will operate in a pulsed mode (according to the current EU reference configuration with a pulse length of $2 \mathrm{~h}$ ) [6].

- Fuel injection is able to take a mixed deuteriumtritium input rather than separate isotopic feeds, negating the need for isotope separation for this purpose. 
- The tritium plant will consist of as few systems as possible - to reduce cost and complexity and ensure reliability.

- Minimisation of tritium inventory is a prime requirement. This strongly prefers continuous technologies over batch processes, wherever possible and utilizes the idea of continuous Direct Internal Recycling (DIR) [7], which introduces shortcuts within the fuel cycle directly from exhaust to fuelling, thus, avoiding complete D-T separation of the fusion exhaust gas.

- There is a trade-off between the cost of re-use of plasma enhancement gas (PEG) and the cost of purchase in case the PEGs are dumped, together with increased loading on exhaust detritiation.

This paper is an overview paper with more detailed publications on the individual sub-systems to come. The paper will delineate in a staged systems engineering approach the content of a coherent $\mathrm{R} \& \mathrm{D}$ programme for the complete inner fuel cycle of DEMO, separately for the three sub-systems. Once the requirements are determined, functional needs will be derived that would fulfil the requirements. In a follow-up step, technologies are screened that have the potential to fulfil the required functions. In a last step, the technologies were then ranked against specific criteria to finally identify the ones which are 'best' suited, and also, to reveal existing gaps on the way to DEMO, considering already existing R\&D for ITER. The outcome of these analyses will then be taken as the basis to define specific $R \& D$ actions for the DEMO inner fuel cycle development within the Work Package TFV (Tritium, Matter injection and Vacuum) of the EUROfusion Consortium.

\section{Functional break-down of the inner fuel cycle}

The fuel cycle on an abstract level is seen as a system that has to provide certain functions to fulfil certain user requirements. This is illustrated in Fig. 1 that highlights the chain and associated growth of details on the systems engineering path from top to bottom. It is important to note that in our approach functions follow requirements and technologies follow functions. Consequently, to start from an existing plant break-down structure and plant architecture, such as for ITER, and to 'bend' it to fulfil a functional break-down structure for DEMO is in our understanding incorrect.

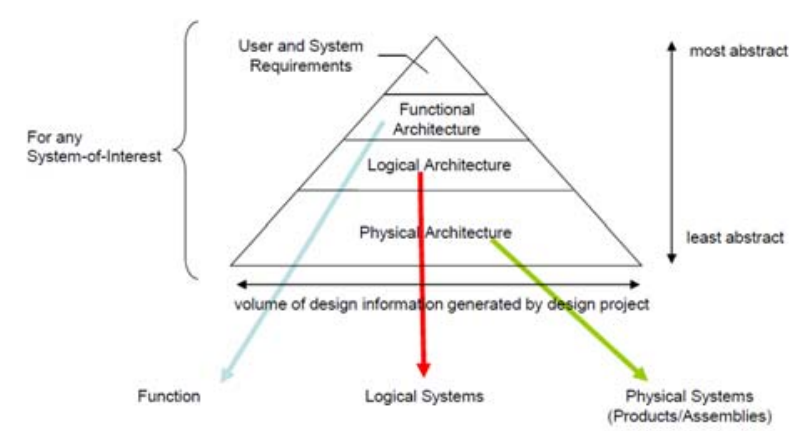

Fig. 1. Systems development logic (modified from [8]).
In the following, we address the functional breakdown structure (FBS) of the sub-systems (see also [9, 10]).

An integral function of the fuel cycle is to ensure safety by (i) confining radioactivity (to confine source terms, to confine and detritiate tritiated gases) and (ii) limiting exposure to ionizing radiation. Another integral function of the DEMO fuel cycle is to support economic attractiveness of the produced electric energy by (i) providing a sufficiently good RAMI performance (Reliability-Availability-Maintainability-Inspectability), (ii) providing a sufficiently short dwell time, and (iii) reducing capital expenditure (CapEx) and operational cost (OpEx). Furthermore, it is an integral function to protect investment of the DEMO machine, and to help to satisfy the stakeholder and DEMO user requirements by appropriate translation of these to what it means for the fuel cycle.

The function of the tritium plant is to (i) supply hydrogen isotopes to the machine, (ii) treat, store and supply tritium gas, (iii) to manage gas intake from the tritium extraction system of the breeding blankets, (iv) to provide purification (tritium recovery) of the breeding blanket coolants, and (v) to recover tritium from the exhaust detritiation system, maintenance activities and tritiated waste.

The function of the matter injection system is to ensure plasma burn operation by (i) enabling plasma density ramp-up and maintaining core density by matter injection, (ii) mitigating ELMs and disruptions, (iii) providing plasma enhancement gases (radiative seeding, metal wall confinement recovery), and to ensure plasma dwell operation by injection of support gases such as hydrogen or helium for glow discharge cleaning and wall conditioning.

Finally, the function of the pumping system is to ensure plasma burn operation by (i) helium ash exhaust, by (ii) pump-out of unburnt fuel at a rate and pressure so that divertor detachment can be sustained, and (iii) by maintaining the required vacua of the diagnostics, service and heating systems. Plasma dwell operation must be ensured by providing the necessary dwell pressure, and machine operation has to be supported in any other non-burn phase (wall conditioning, ultimate pump-down after interventions etc.).

We have performed a functional analysis first, which is completely solution-neutral und un-biased by preconceived ideas, followed by a technology survey which is completely solution-specific. To have an unbiased approach in finding the 'best' technology, we developed a list of criteria which were then ranked by a procedure called pairwise comparison to weight the criteria for application to technology solutions candidates. This was also done to make sure that the $\mathrm{R} \& \mathrm{D}$ programme is strictly driven by needs of the DEMO plant design and not primarily by scientific challenges as such. In a final step to define the most efficient R\&D programme, we added existing managerial and resource constraints. 


\subsection{Tritium functional architecture}

To meet the FBS above, the tritium plant is given the following capabilities:

- An intake gas processing capability for vacuum pumping exhaust gas, the outputs of which are three separate streams comprising (i) a mixed purified tritium/deuterium stream for direct reinjection into the tokamak, (ii) a separate purified stream of mixed PEGs (potentially separated from DT) to be further separated into the different gas species for reinjection into the tokamak, and (iii) a waste stream consisting of helium isotopes and other impurities that have entered the tokamak (carbon mon/dioxide, ammonia, etc.)

- An intake and supply capability for tritium gas ultimately fed from the breeder blankets.

- A deuterium supply.

- A gas distribution and control capability which regulates the supply of gases for injection into the tokomak.

The ratio of deuterium to tritium injected into the tokamak is maintained by changing the ratios of the flow rates of re-injected recirculated mixed deuterium-tritium gases and discrete deuterium and tritium feeds. This should compensate fuel burn-up, tritium decay, and any imbalances caused by isotope separation effects within plant sub-systems. Thus, the central idea to meet the key objective of minimised inventory in the tritium plant is that under normal steady state operational conditions, there is no general storage or release of gases (other than impurities and helium isotopes), and no attempt to provide full isotopic separation within the main loop. To achieve fine-adjustment of hydrogen isotope compositions, protium $\left(=\mathrm{H}_{2}\right)$ removal and some isotope rebalancing will be included in the design. To accommodate reactor start-up/shut-down and also pulse operation, additional capabilities are needed, in particular storage for mixed deuterium-tritium and PEGs. A switchable recirculation path is provided between the gas distribution sub-system and the pumping system, bypassing the tokamak, to allow an appropriate volume of fuel to be made available from the storage facilities in advance of tokamak start-up. This allows for the possibility of slow release of fuel from the storage facility upon tokamak start-up and to accommodate pulse mode operations. It also provides an additional capability for rapid capture and safe storage of tokamak gases under emergency conditions.

To complete the architecture, an isotope rebalancing system is placed in series with the DT output from the exhaust processing system. This is required to accommodate isotopic effects in other system blocks, and to allow some necessary degree of control of the D:T ratio in the fusion reaction [11]. The fraction of gas rejected from the isotope rebalancing system is fed to the isotope storage system. Furthermore, a protium removal stage is included as a bleed off from the primary tritium loop, in order to keep protium levels low. It is anticipated that protium will appear in the exhaust stream as a result of the ingress of water and from outgassing from the main tokamak vacuum vessel. The waste stream from this process is directed to the exhaust detritiation facility, which removes tritium and minimises the tritium release to the environment.

An additional purification function is needed to recover tritium from coolant loops in the breeder and power plants, sending it to downstream processing. A second function is to control the coolant chemistry.

\subsection{Matter injection functional architecture}

Based on the considerations above, we have given all gas provision functions (supply of all gases, not only hydrogen isotopes, at the required purity and composition and of the required flowrate) to the tritium plant. This is functionally very similar to the situation at ITER. Hence, the matter injection systems function is limited to process an inlet gas stream coming from the tritium plant or via the DIR path, and to inject the matter in gaseous or in frozen form such that the FBS is set.

\subsection{Vacuum functional architecture}

Several vacuum systems are needed to operate a fusion power plant. To meet the FBS above, the vacuum system is given the following capabilities:

- An intake processing capability for gas coming via the divertor at the full vacuum range (burn and dwell conditions), resulting in a simple linear arrangement of primary and rough vacuum pumps with gas entry points upstream and in-between.

- A vacuum provision capability for service, heating (e.g. neutral beams) and auxiliary systems (e.g. cryostat) (not necessarily tritiated).

- A vacuum provision capability for the tritium extraction systems from the breeding blankets which are expected to work under vacuum (vacuum sieve tray or permeation against vacuum).

The ITER gas exhaust and cleaning system is based on the philosophy to transfer the full amount of exhaust gas via primary and rough vacuum pumps to the tritium plant, and there to separate the hydrogenic exhaust gas mixture to pure isotopes in a defined way so that the wanted fuel mixture can be mixed up for re-injection into the torus [12]. For DEMO, we take benefit from the fact that the fuel will always be injected at approximately the same DT ratio, so that a gas mixture of an appropriate composition which would be separated from impurities and helium isotopes within the vacuum system, would allow immediate re-cycling and reduced flows of gas to be processed in the tritium plant. Obviously, this will work only, if the separation factor is sufficiently high and if there is a feedback controlled fuel gas management which is able to produce the correct composition from various source flows. This concept of Direct Internal Recycling shall be utilized in the DEMO inner fuel cycle [7].

The resulting fuel cycle architecture in simplified form is shown in Fig. 2. 


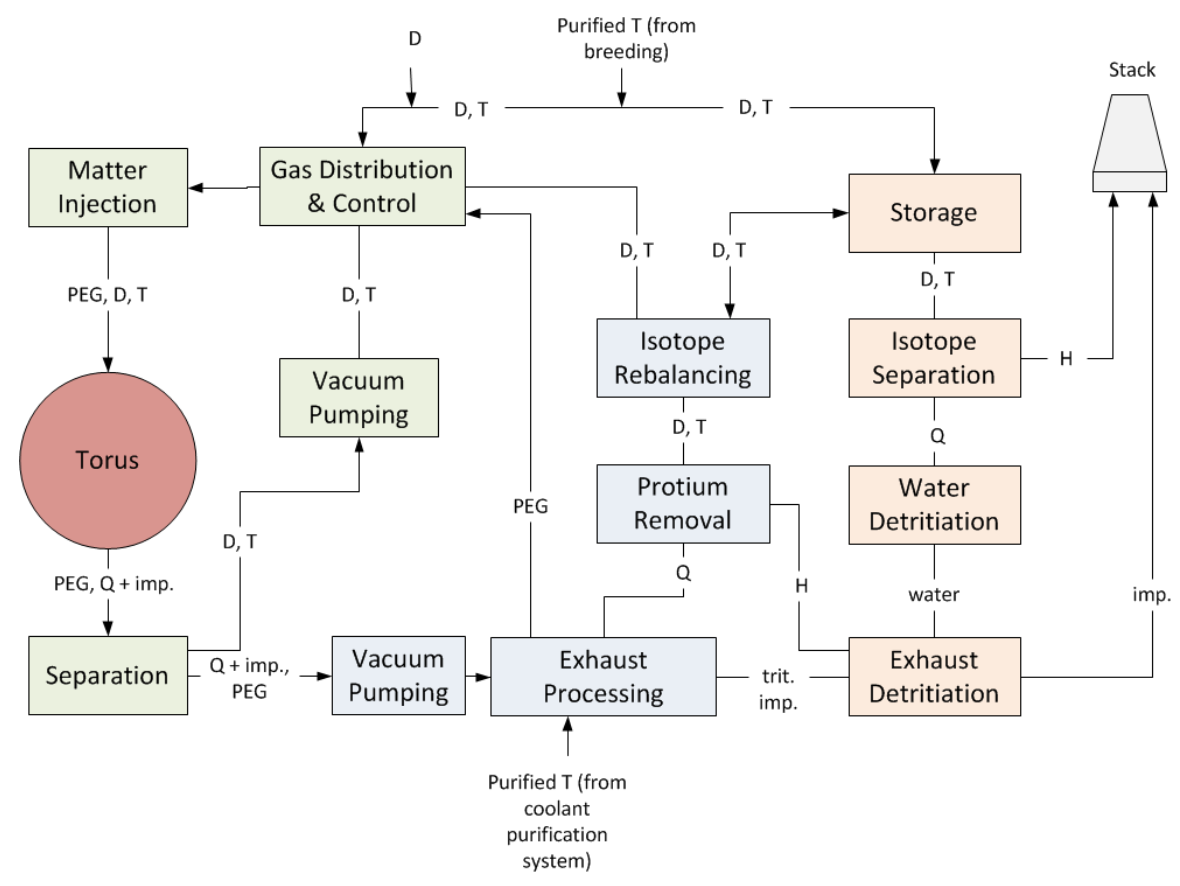

Fig. 2. DEMO fuel cycle architecture (simplified). Q stands for any of the hydrogen isotopes $\mathrm{H} / \mathrm{D} / \mathrm{T}$, imp. denotes impurities.

The figure illustrates the new loop concept to reduce inventories, with a direct internal recycling loop and separation close to the torus, with a continuous main tritium plant loop for re-injection and an outer tritium plant loop.

\section{Technology review and gap analysis}

This step performs a survey of potential technologies to provide the named functions above followed by an optioneering approach to sort the found technology candidates for each functional system block in a quantitative manner. This process comprises:

(i) Identifying generic selection criteria.

(ii) Refinement of these criteria to system blocks on an individual basis, i.e. the development of specific selection criteria for each system block.

(iii) Determining the relevance of the selection criteria for each system block using the Pairwise comparison (Pugh matrix) approach [13] for weighting the criteria accordingly. This approach is considered to be most useful to prevent subjective selection of weightings or deliberate manipulation of weightings to skew results.

(iv) Scoring each technology candidate for a system block against each selection criteria. The scoring how a certain technology solution fulfils the criteria was from 0 to 5 - unsatisfactory to very good.

(v) Applying the weighting factors determined from the Pairs analysis method for the selection criteria to develop a ranked set of technology candidates for each functional system block [14].

The core selection criteria to be applied to all system blocks fall into the categories as shown in Table 1 . The following sections explain the optioneering results and state the R\&D needs arising from that. Table 2 at the very end of this chapter 3 presents the numbers behind the selected prime technologies and compares the results of the three sub-systems with each other.

Table 1. Used generic selection criteria in the technology review.

\begin{tabular}{|c|c|}
\hline Category & Generic interpretation \\
\hline \multirow{4}{*}{ CapEx } & Low development effort/costs \\
\hline & Low manufacturing costs \\
\hline & $\begin{array}{l}\text { Low infrastructure } \\
\text { requirements }\end{array}$ \\
\hline & Compatibility with other plant \\
\hline \multirow{5}{*}{ OpEx } & Low total cost of ownership \\
\hline & Low operational efforts \\
\hline & Low maintenance requirements \\
\hline & Good maintainability \\
\hline & Low power consumption \\
\hline \multirow{4}{*}{$\begin{array}{l}\text { Performance/fit } \\
\text { for purpose }\end{array}$} & $\begin{array}{l}\text { High functional and range } \\
\text { flexibility }\end{array}$ \\
\hline & High throughput \\
\hline & High accuracy/precision/purity \\
\hline & $\begin{array}{l}\text { High performance and } \\
\text { efficiency/fit for purpose }\end{array}$ \\
\hline Risk & $\begin{array}{l}\text { Low development risk, similar } \\
\text { solution exploited elsewhere } \\
\text { (e.g. at ITER), reliability }\end{array}$ \\
\hline \multirow{4}{*}{$\begin{array}{c}\text { Safety \& } \\
\text { environment }\end{array}$} & Low tritium inventory \\
\hline & High safety/low hazards \\
\hline & Tritium compatibility \\
\hline & Environmental acceptability \\
\hline Operation type & $\begin{array}{l}\text { Continuous preferred over } \\
\text { batch }\end{array}$ \\
\hline $\begin{array}{c}\text { Technology } \\
\text { readiness }\end{array}$ & System maturity \\
\hline
\end{tabular}




\subsection{Tritium technology review}

Exhaust processing. The main function of the exhaust processing system is the separation of the deuterium/tritium stream for re-injection into the tokamak; a purified stream of PEGs will also be produced as well as a waste stream of helium isotopes and other impurities. Usually, the exhaust processing contains two steps: First, the impurities are removed from the unburnt deuterium/tritium, second, the impurities previously separated are processed to recover tritium.

Four technologies have been screened for impurity removal: (i) cryogenic adsorption [15], (ii) chemisorption in getter beds [16], (iii) palladium membrane technology to allow for DT permeation [17], and (iv) cryogenic freezing [18]. From the results of the technology scoring, the Pd-Ag permeator has obtained clearly the highest score (with 82 out of theoretically $100 \%$ for a technology perfectly fitting all criteria, that means given a score of 5 in all criteria), with cryogenic adsorption or getter beds as batch-wise fall-back solution (with about 70\%). The front-end permeator technology [19] is also used at ITER, therefore important and additional information about this technology can be envisaged from its operation in ITER. At this stage, two main differences arise between the ITER and DEMO impurity separation process: one is related to the gas throughput to be processed and the other one is related to the possible high concentration of PEG in the exhaust gas stream (quantities are not confirmed but PEG concentrations could be higher than 20\%). Theoretically, the higher gas throughput (due to the higher size of DEMO compared to ITER) does not represent a big issue for the $\mathrm{Pd}-\mathrm{Ag}$ permeator technology, since the system can be easily scaled up; but the high concentration of PEG can become an issue. Importantly, the presence of PEGs in the exhaust stream reduces the hydrogen partial pressure on the input side of the permeator and therefore the overall performance of the membrane. In this case several solutions have to be analysed, such as a higher total inlet pressure of exhaust gases, a different membrane design to reduce the Pd-Ag layer, or systems to remove PEG in a separate stage before the impurity separation process ( $R \& D$ topic 1$)$.

The main function of impurity processing is to process the tritiated impurities, previously separated in the impurity removal, for tritium recovery. Methods fall into two categories: Those that use water as an intermediate step (catalytic oxidation of the impurity stream and subsequent treatment by hot metal beds, water-gas-shift reaction and/or electrolysis) and those that do not. With regard to the safety and complexity aspect, a process that avoids formation of HTO as intermediate step is preferred due to the high radiological hazard of HTO. In the area of processes without forming water, the palladium-based membrane reactor technology [20] has obtained the highest score in the corresponding ranking exercise (74\%). In the ITER torus exhaust processing unit, impurity processing is expected to be realized using a multistep process that combines a catalytic bed for hydrocarbon cracking and water gas shift, a Pd-Ag permeator and a membrane reactor (PERMCAT) for final cleaning [21]. Useful information will arise during ITER operation. Some differences between the ITER and DEMO impurity processes come from differences in the impurity compositions to be treated. ITER impurities mainly consist of $\mathrm{Q}_{2} \mathrm{O}, \mathrm{CQ}_{4}$, $\mathrm{He}, \mathrm{CO}, \mathrm{CO}_{2}$ and $\mathrm{NQ}_{3}(\mathrm{Q}=\mathrm{H}, \mathrm{D}, \mathrm{T})$. DEMO will be a fully tungsten machine (i.e. no carbon present), therefore very few (almost zero) tritiated hydrocarbons are expected, and there are no relevant tritium-tungsten chemical reactions.

PEGs are expected to play a major role in tritium system development. However, it is not clearly defined at the moment, which species at which flowrates are to be expected. The current EU DEMO scenario foresees xenon for radiative seeding, nitrogen for confinement recovery, and potentially neon for disruption mitigation by massive gas injection. Once the role of the PEGS has become clearer, the system assessment has to be revisited in all aspects (incl. activation aspects and the need for radioactive waste management functions, as identified in the tritium plant FBS) ( $R \& D$ topic 2$)$.

Already for ITER, R\&D is ongoing to develop more efficient catalysts or alternatives to counter-current isotopic swamping via a protium purge gas stream as utilized in the PERMCAT stage, in order to reduce the flowrates involved. Also for DEMO, in the area of the breeding blanket systems similar functions have to be fulfilled [22, 23]. It is definitely an area of synergy to integrate the various $\mathrm{R} \& \mathrm{D}$ activities in this field $(R \& D$ topic 3).

Main loop protium removal and isotope re-balancing. As a consequence of the continuous flow approach in the main loop, one needs a technology to remove the protium fraction. There exist a great number of hydrogen isotope separation technologies which have been studied carefully. In line with what was said above, we try to avoid technologies that are associated with water in the main loop, for energy consumption (operational cost), safety and inventory reasons. In consequence, cryogenic distillation, which is a well-established technique that has been used for many decades for isotope separation, also in ITER (together with the combined electrolysis and catalytic exchange process [24]), and could likely be adapted to DEMO did only reach a score here of $64 \%$ (it will be revisited for exhaust detritiation functions in the outer loop). Furthermore, some more exotic, potentially promising high-purity separation technologies were found (such as plasma separation [25], laser isotope separation [26] or cryogenic quantum sieving [27]), but they were considered to be a too high risk option since there does not appear to be sufficient literature to demonstrate that they work on hydrogen isotopes and in technical scale.

The technology that was decided to become baseline was the Thermal Cycling Adsorption Process (TCAP) with a score of $72 \%$. TCAP is a chromatographic process, but, contrary to the classical batch chromatographic sorption column is operated in semi- 
continuous manner in a thermal cycling pattern which allows continuous operation after an initial conditioning phase. The TCAP concept has been fully validated experimentally in different designs at SRNL, USA [28] and CEA Valduc, France [29], although operation at DEMO scale would be unique and utilizing it for protium is more demanding than for the heavier isotopes ( $R \& D$ topic 4$)$. Even if combined here in one function, it has to be noted that for protium removal, the purity requirements are clearly higher than for re-balancing so that different technologies may be utilized in future stages of the design development ( $R \& D$ topic 5$)$.

Tritium and mixed hydrogen isotope storage. Hydrogen storage technologies exploit physi- or chemisorption on solids. For decades and with very little modification over the last 20 years, the preferred method of tritium storage is on depleted uranium beds, as they exhibit almost ideal pressure and temperature hydrogenation parameters. However, while adequate for small-scale application, it does not scale proportionately with the quantity of tritium being stored. Due to the large bed expansion upon tritide formation, as well as the need for cooling during tritium absorption, only relatively small U-beds are suitable. Hence, a large tritium inventory would require many parallel uranium beds. Furthermore, regulatory restrictions surrounding the use of uranium as a controlled nuclear material mean significant additional effort to its use.

This is why, in an alternative evaluation, a modified criteria list was set up, where the criterion 'non-nuclear material' was introduced, so that U-beds rank lower, and the technology readiness criterion was neglected. In this exercise, the storage material with greatest development potential (score 63\%) was found to be with magnesium hydride, ball-milled with $1 \mathrm{~mol} \% \mathrm{Nb}_{2} \mathrm{O}_{5}$ [30], but developing this material into a full tritium storage system will take years of design and improvement. The use of Zr-Co was another candidate solution (score of 55\%), which has already been widely investigated in the past, as it was also seen as an option for ITER, before being discarded mainly due to RAMI and significantly less perfect hydrogenation parameters [31].

In consequence, it is felt that tritium storage in a future larger tritium plant could be achieved through existing technology. However without developments in this area, storage could become a limiting process step within the operation of the plant by imposing limits on the functional operation of the reactor. Hydrogen storage technologies are also being developed in other disciplines, and applying these developing technologies to tritium storage would hopefully produce a solution to the current concerns. The social pressure on the use of nuclear licensed materials poses a risk to storage in a future tritium plant. It is feasible that uranium may be forbidden by some governments in countries wanting to employ fusion power. Therefore developing an alternative which is both based on non-nuclear materials and could outperform existing technology would be ideal ( $R \& D$ topic 6$)$.
Blanket coolant purification. This task is characterized by the tritium permeation rate out of the blanket, the purification tritium removal efficiency, and the allowable tritium activity in the coolant. Considering that the coolant could be either helium or water, the technology scoring has to be performed separately for helium and water coolant loop tritium extraction.

In the case of helium, tritium removal is a very delicate process because of the large helium flow rate to be treated in which low $\mathrm{Q}_{2} \mathrm{O}$ and $\mathrm{Q}_{2}$ molar fractions and small quantities of impurities are contained. At this stage, there is a large uncertainty about the amount of tritium in the helium coolant, which depends on many aspects, above all the use of permeation barriers and their relative permeation reduction factors. Conversely to exhaust processing, for the coolant purification system, a simple scale up of the system from ITER-TBM to DEMO would not be possible. In fact, even if only a small fraction (less than 1\%) of the coolant will be processed inside the coolant purification system, the difference between the amount of coolant in ITER and DEMO is very large (factors in the order $10^{3}$ to $10^{4}$ ) [32]. Chemi- and physisorption beds (cold traps) in quasi-continuous pressure swing mode have received the highest score (around 70\%) especially in view of their possibility to treat large quantities of gas. Research activities to improve the reliability of sieving materials (i.e. zeolite) and the performance of particular getter beds (heated getter bed) are ongoing ( $R \& D$ topic 7 ). Eventually, both technologies have to be integrated with an oxidizing bed [33].

Also for blanket concepts with water as coolant, the tritium permeation into cooling water is strongly affected by the operating conditions (P, T, water chemistry) and the effectiveness of the permeation barriers. For the WCLL (water-cooled lithium-lead) concept, the low breeder velocity in the blanket and the low tritium solubility in lithium-lead can lead to a significant tritium activity in the liquid metal and consequent permeation. Therefore, the permeated tritium has to be recovered for safety and economic reasons. Tritium extraction from water is a difficult task with significant impact on plant size and cost ( $R \& D$ topic 8$)$. In ITER, tritium removal from water is performed in the water detritiation system (WDS) by means of the CECE process (combination of electrolysis and catalytic exchange) [24] which reduces the total amount of tritiated water via tritium enrichment at the electrolyser and at the same time achieves decontamination factors of several orders of magnitude along the liquid phase catalytic exchange column [34]. At this stage, the CECE process (having reached a score of $65 \%$ ) appears the best choice also for the DEMO water coolant purification system even if main drawbacks (like investment cost for platinum catalyst, operating cost for the electrolysers, and explosion risk with electrolysis) have to be overcome in view of a larger scale plant. However, also in this case further information will be available during the CECE operation in ITER and from existing large scale plants for heavy water reactors. 
Exhaust detritiation. The most common technology for exhaust detritiation is the catalytic conversion of the hydrogen isotopes to water by means of recombiners (one operating at low and one operating at high temperature) and subsequent adsorption of the formed water to be sent to WDS. ITER will use scrubber columns where operation is based on isotopic exchange between tritiated water vapour and tritium-free liquid water. The main advantage over the previously considered sieve beds is that no regeneration cycle is needed and the system is more compact [35]. Useful information about this technology will arise during ITER operation and from a Japanese pilot plant.

Gas distribution control, tritium tracking and accountancy. As delineated above, one of the core new features of the DEMO plant is continuous operation avoiding the use of intermediate storage wherever possible. This of course requires a capable online and real time system of tritium measurements (as will also be required for tritium accountancy), which will clearly be beyond the current state-of-the-art, eventually demanding rather sophisticated control algorithms (R\&D topic 9).

\subsection{Matter injection technology review}

A number of matter injection technologies have been studied, including conventional gas puffing, supersonic gas injection and pellet injection for various velocity ranges. Classical (gas gun type or centrifuge driven) pellet injection for low and medium velocities $(\mathrm{km} / \mathrm{s}$ range) [36], microwave [37], laser ablation [38] and railgun pellet injection (elevated velocities, $10 \mathrm{~km} / \mathrm{s}$ range) as well as compact tori [39] or unmagnetized plasma injection [40] for high velocities $(100 \mathrm{~km} / \mathrm{s}$ range).

In translating the generic selection criteria to specific ones for matter injection, groups formed at three different levels of relevance were found, with reliability, fuelling efficiency and tritium inventory being ranked as most essential. Finally, classical pellet injection was found to be the prime candidate for the DEMO core fuelling system (scoring 75\%), being the only one with no zero score in any of the ranking criteria. Pellet fuelling relies on the injection of mm-sized bodies of solid ice formed from hydrogen isotopes. For the sake of high fuelling efficiency, it is believed the launch has to be made at velocities of at least $1 \mathrm{~km} / \mathrm{s}$ through guiding tubes from the torus inboard side [41]. Acceleration takes place using conventional pellet launchers; these are blower guns, gas guns (single or multi stage) and mechanical centrifuge devices. In the future, the question arises regarding the need for additional R\&D efforts required to close a possibly still emerging gap with respect to reactor needs even once a capable ITER system, which is also based on conventional pellet injection, is developed. It is considered that since ITER decided to adopt classical pellet injection the gap between that available and technology needed for DEMO has become even larger, particularly as R\&D has subsequently been limited to ITER support only.
$R \& D$ in the matter injection area will be defined by the results of pellet transport and ablation modelling studies which translate the DEMO plasma scenario in pellet injector engineering requirements (parameters such as e.g. launch location and according injection velocity, optimized pellet mass flow for according pellet size and rate) ( $R \& D$ topic 10 ) [42]. Potential needs are outlined in the following sections, separately for the three sub-systems of a typical pellet launching system.

Pellet source. Capable and reliable steady state extruders are already employed in several operational pellet systems, for example on JET (High Frequency Pellet Injector HFPI [43]) or in the 1:5 scale prototype of the ITER extruder [36]. Well suited for their actual missions, for DEMO these systems still miss e.g. the capability of $\mathrm{T}$ processing, operation periods in the hour or day regime and measures for efficient fuel recovery. The development of a full scale extruder for ITER is reported to be progressing well. Hence, as long as the ITER efforts on this topic are ongoing successfully, no extra R\&D activity seems to be required for DEMO.

Pellet accelerator. A similar situation exists for the development of an appropriate pellet launcher as long as the speed range of blower guns is considered, covering pellet speeds up to $300-500 \mathrm{~m} / \mathrm{s}$. In case higher speeds are envisaged, as is the case for DEMO, the situation is essentially different and additional RD is needed. For single stage guns, operation at sufficient rates at speeds in the range at and above $1 \mathrm{~km} / \mathrm{s}$ for long periods has to be established. In order to gain more headroom with respect to pellet speed, further development of multi stage gas guns would be beneficial [44]. JT-60SA could serve as a proper test bed since first considerations on the pellet fuelling system indicate the need of a proper high speed launcher, most probably a multi stage gas gun, in order to go for a low field side straight injection path due to the fact that the injection track towards the high field side is not possible because of space restrictions. Mechanical centrifuge accelerators can cope with high repetition rates, but no operation has been yet demonstrated with continuously working extrusion systems and long pulse lengths for sufficient rates. Hence, additional R\&D efforts are required dedicated to the operation under steady state conditions for suitable long periods, preferably with pellet feed in from multiple extruders in parallel. Furthermore, the compatibility with requirements for operation with $\mathrm{T}$ and improvements making still higher launch velocities possible are worthwhile $\mathrm{R} \& \mathrm{D}$ targets ( $R \& D$ topic 11 ).

Pellet guiding system. For the three major system components, the largest gap seems to exist for the transfer system. Although it seems obvious such a system is needed for efficient particle fuelling and also to minimize transport losses forming a severe potential load on pumping and fuel recovery systems, no dedicated R\&D is under way right now and very little has been invested in the past, also not in the ITER program. Construction of the high speed transfer system at AUG was done in a heuristic way, suitable for basic fuelling needs of a medium size tokamak but never fully 
optimized. The same approach was chosen for JET, where the former dissatisfactory transfer system laid was revised; in its rearranged set up, the JET HFPI transfer system will be dedicated solely for inboard pellet launch with high reliability and rates up to about $30-50 \mathrm{~Hz}$ [43]. However, due to the limitation of the applied acceleration system it cannot be tested at high transfer speeds. It is therefore suggested to conduct additional detailed investigations in order to analyse the influence of the guiding tube geometry - both with respect to the radius of curvature but also to the tube cross section and the pellet mass and shape on the maximum speed at which a solid pellet can transfer without fracturing. Further on, the impact of the pellet ice parameters like temperature and hydrogen isotope composition (including the potential of admixed pellets [45]) and eventually the processing of the ice formation on maximum transfer speed but also mass transfer loss deserves further attention. Accordingly, efforts devoted to potential DEMO needs would be best performed in a test bed laid out in line with the expected DEMO parameter regime ( $R \& D$ topic 12$)$.

Injection of PEGs will be based on gas puffing with appropriate shutters, which can largely benefit from R\&D for ITER.

\subsection{Vacuum technology review}

The analysis was done based on the well-known vacuum family tree [46] which covers both primary and roughing pump technologies. The results of this analysis are only given in short for completeness here, full details have been published elsewhere ([47], the scoring exercise has been re-worked to be consistent with the criteria of Table 1).

Primary Pumps. The application of high vacuum pumps during burn in fusion is very unusual in such a sense that it asks for high pumping speeds $\mathrm{S}$ due to the high gas flows $\mathrm{Q}$ to be processed, whereas in most other applications (also during the dwell phase), high pumping speeds do normally result from the very low pressure requirements the pumps are asked to achieve $(\mathrm{S}=\mathrm{Q} / \mathrm{p})$. Having said that, all primary pump technologies that do only provide the requested pumping speed and capacity at very low pressures (such as sublimation pumps and ion pumps) are not appropriate. In summary, three pump technologies have been identified as at least partly suitable:

- Cryogenic pumping (score of 50\%): The standard pump technology used on modern medium-size tokamaks could be an option but require significant R\&D to implement a hydrogen isotope separation function for the DIR [48, 49] (R\&D topic 13). However, cryogenic pumps have high energy requirements (the ITER cryopumps require 25-30\% of the cryo-plant capacity), cryogenic safety issues (rapid expansion of collected gases upon failure, and importantly, result in high inventories of retained tritium between regenerations.

- Metal foil pumps (70\%): Metal foil pumping is a technology that is not (yet) used commercially $(R \& D$ topic 14). It exploits the principle of superpermeation [50]. This makes use of the fact that metal foils can show a high permeability for hydrogen atoms at a certain atomic energy, which recombine to form molecules at the back side of the foil. This pump works only for hydrogen isotopes as a pump; it is not applicable for pumping helium and other heavy gases. So, a metal foil pump can ideally be used for separating and compressing the hydrogenic exhaust gas fraction close to the torus which then could be recycled directly to the fuelling systems for reinjection. Only the remaining helium and impurity enriched part of the gas would then need to be treated in the tritium plant. The metal foil pump is seen as the perfect solution to implement DIR already in the vacuum pumping part of the fuel cycle.

- Vapor diffusion pumps (70\%): Diffusion pump technology is well-known, including successful application to pumping tritium (in this case, a tritiumcompatible working fluid such as mercury is required) [51, 52], but the applicability for high throughputs requiring jet stages to be integrated is novel ( $R \& D$ topic 15$)$.

Roughing Pumps. The most challenging requirements for mechanical roughing pumps for DEMO are the required tritium compatibility and corresponding sealing needs for tritium containment.

There are commercial tritium-compatible pumps in use: metal bellows pumps, piston pumps and scroll pumps [53]. To address the tritium-oil/lubricant interaction issue they are made in a 'dry' (oil-free) design. But to achieve sufficient lifetime, the tolerances have to be increased which results in relatively moderate ultimate pressures (especially for hydrogen) and only small pumping speeds. In addition, the reliability of these pumps has always been a problem. The technology survey showed that dry reciprocating positive displacement pumps are not a preferred solution (with scores of around $70 \%$ ), mainly due to maintenance and poor performance aspects. Whereas the liquid ring pump as most suited representative of the rotary positive displacement vacuum pumps would be most promising (scoring 91\%), if a tritium-compatible working fluid would be found ( $R \& D$ topic 16 ).

This recommendation resulted in a $\mathrm{R} \& \mathrm{D}$ programme to develop a mercury based liquid ring pump together with a mercury vapour diffusion pump, and a metal foil pump to implement the DIR function [54]. The mercury pump configuration is also usable for the neutral beam injection vacuum system. But here, an alternative technology based on new high capacity getter materials is under study as well.

Similarly as in the matter injection sub-project, the divertor pumping system via the sub-divertor area has a very strong interface with the plasma and has also the function of a (slow) actuator for plasma control. This is why an extensive R\&D activity is under way to develop tools which translate physics parameters into engineering 
parameters (contour calculations of pressures and density, capture coefficients) $[55,56]$ (R\&D topic 17$)$.

\subsection{Review summary}

Table 2 presents under each function the weightings that were obtained for the criteria according to Table 1 , together with the individual scoring results (from 0 to 5 ) for the technology choices which require additional $\mathrm{R} \& \mathrm{D}$. It is very convincing that all selected candidates have achieved scores between $65 \%$ and $90 \%$, which is an impressive demonstration of the successful work of past world-wide fusion programmes. However, it is also revealed that for each of the chosen technologies weak aspects exist (with scores less or equal 3). The future R\&D programme can now be tailored to focus on exactly these areas. This illustrates one of the advantages of the chosen system engineering approach, namely to identify a solution not as total, but in a diverse way.

One criticism to this approach is that the weighting exercise is subjective and can be biased. In order to address this point, the weightings have been discussed in detail in expert groups (comprising experienced senior scientists and advocates for specific solutions). It has been found that, although criteria weightings and technology scorings could differ, the final outcome did not change and is therefore considered to be very robust. It was also so, that in most cases, second and third choices had quite a distance (15-20\% to the first choice solution).

\section{Definition of a consistent $R \& D$ programme}

The gap analysis as described above was now studied in detail to tailor an efficient programme for the European contribution to R\&D for the inner fuel cycle.

Table 2. Quantitative optioneering table for the DEMO inner fuel cycle prime technologies and R\&D programme.
It is believed that this has been done for the first time in such an integrated manner. When defining an efficient R\&D programme, a major point was to review ITER technologies for their applicability to delivering the functionality required for each DEMO tritium plant system block. There is a strong argument to utilize ITER experience and exploit the development work done there. Besides the general objective to minimize inventory (and hence to prefer continuous processes), the following three factors have been considered when assessing whether a technology available via ITER is applicable to a DEMO reactor:

- Function - does the system perform the necessary processes applicable to a DEMO reactor?

- Proven technology - if an ITER technology is not mature or applicable to DEMO, then it has no advantage over that which might arise from DEMO $\mathrm{R} \& \mathrm{D}$

- Scale - could the system process feasibly the required throughputs?

Based on the above, the major R\&D headlines as summarized in Table 3 have been implemented in the EUROfusion programme. In developing this, existing additional resource constraints have been considered.

The system-specific R\&D actions will be accompanied by two more action lines, which are only mentioned for completeness here. One is the development of a fuel cycle simulator that supports design optimisation and sensitivity analyses to identify areas with largest impact potential. Strongly linked to that is the development of an encompassing concept for tritium accountancy.

It is noted that the application of the decisionmaking scheme above did not pose any problems. It provides a documentation that in the future, if the boundary conditions for the programme change, decisions can be modified in a logical and defendable manner. To this end, it is suggested to use this approach further and expand to other areas of DEMO, for example to combine inner and outer fuel cycle.

\begin{tabular}{|c|c|c|c|c|c|c|c|c|c|c|c|c|c|c|c|}
\hline Function & $\begin{array}{c}\text { Exhaust } \\
\text { Processing }\end{array}$ & & & $\begin{array}{l}\text { Protium } \\
\text { removal }\end{array}$ & & $\begin{array}{c}\text { Coolant } \\
\text { purification }\end{array}$ & & & $\begin{array}{c}\text { Core } \\
\text { fuelling }\end{array}$ & & $\begin{array}{l}\text { Primary } \\
\text { Pumping }\end{array}$ & & & $\begin{array}{c}\text { Rough } \\
\text { Pumping }\end{array}$ & \\
\hline Technology & & $\begin{array}{c}\text { Permeator } \\
\text { for } \\
\text { Impurity } \\
\text { Separation }\end{array}$ & $\begin{array}{c}\text { Membrane } \\
\text { reactor for } \\
\text { Impurity } \\
\text { Processing }\end{array}$ & & TCAP & & $\begin{array}{c}\text { Sorption } \\
\text { bed for } \\
\text { He } \\
\text { coolant }\end{array}$ & $\begin{array}{c}\text { CECE } \\
\text { for } \\
\text { water } \\
\text { coolant }\end{array}$ & & $\begin{array}{c}\text { Pellet } \\
\text { injectio } \\
\mathrm{n}\end{array}$ & & $\begin{array}{c}\text { Metal } \\
\text { foil }\end{array}$ & $\begin{array}{l}\text { Vapor } \\
\text { diffusion }\end{array}$ & & $\begin{array}{c}\text { Liquid } \\
\text { ring }\end{array}$ \\
\hline \multicolumn{16}{|l|}{ Category } \\
\hline CapEx & 2 & 2 & 3 & 5 & 3 & 3 & 4 & 2 & 6 & 3.5 & 1 & 3 & 3 & 1 & 4 \\
\hline OpEx & 6 & 4 & 4 & 8 & 4 & 9 & 3.5 & 3 & 14 & 4 & 19 & 3 & 3 & 12 & 4 \\
\hline $\begin{array}{l}\text { Performance } \\
\text { /fit for } \\
\text { purpose }\end{array}$ & 5 & 4.5 & 4.5 & 11 & 2.5 & 10 & 4 & 4 & 20 & 3.5 & 17 & 3.5 & 4 & 11 & 4.5 \\
\hline Risk & 11 & 4 & 3 & $l$ & 5 & 12 & 4 & 4 & 23 & 4 & 9 & 3 & 4.5 & 7 & 4.5 \\
\hline $\begin{array}{c}\text { Safety \& } \\
\text { environment }\end{array}$ & 16 & 4.5 & 4 & 16 & 3.5 & 12 & 3.5 & 3.5 & 21 & 3 & 18 & 3 & 4 & 12 & 5 \\
\hline $\begin{array}{c}\text { Operation } \\
\text { type }\end{array}$ & 5 & 4 & 4 & 6 & 4 & 10 & 2 & 3 & 13 & 4 & 16 & 4 & 5 & 13 & 5 \\
\hline $\begin{array}{l}\text { Technology } \\
\text { readiness }\end{array}$ & 3 & 3 & 3 & 3 & 5 & 6 & 4 & 4 & 14 & 4 & 10 & 2 & 4 & 7 & 4 \\
\hline Specific & - & - & - & $\begin{array}{c}\text { High } \\
\text { purity } \\
14\end{array}$ & 4 & $\begin{array}{c}\text { Modify } \\
\text { coolant } \\
\text { chemistry } \\
5\end{array}$ & 2 & 2 & $\begin{array}{c}\text { Transfer } \\
\text { system } \\
\text { okay } \\
23\end{array}$ & 4 & $\begin{array}{c}\text { Gas } \\
\text { separation } \\
\text { possible } \\
18\end{array}$ & 5 & 1 & - & - \\
\hline Score & (of 16$)$ & $82 \%$ & $74 \%$ & (of 18 ) & $72 \%$ & (of 18) & $68 \%$ & $65 \%$ & (of 30) & $75 \%$ & (of 30) & $70 \%$ & $70 \%$ & (of 20$)$ & $91 \%$ \\
\hline
\end{tabular}


Table 3. Implemented DEMO fuel cycle R\&D programme.

\begin{tabular}{ll}
\hline Sub-System & Research area \\
\hline & Specific PEG related R\&D on \\
& permeators and membrane \\
& reactors \\
& TCAP for main loop \\
& requirements at DEMO scale \\
& He coolant purification \\
Tritium & Water coolant purification \\
& Inline and on-time tritium \\
& tracking and accountancy \\
& Control of isotope re-balancing \\
& and gas distribution \\
\hline Hatter & High-speed conventional pellet \\
acceleration \\
Guiding tube transfer system \\
optimization \\
Pellet core fuelling modelling \\
Metal foil pumps based on \\
superpermeable membranes \\
Mercury vapour diffusion \\
pumps \\
Mercury liquid ring pumps \\
Cryopumps with hydrogen \\
separation function for DIR \\
Sub-divertor particle exhaust \\
modelling for divertor \\
optimization \\
High capacity NEG materials \\
\hline \\
Development of a fuel cycle \\
simulator at various levels \\
Consistent DEMO tritium \\
accountancy scheme \\
\hline
\end{tabular}

\section{Status and Outlook}

The TFV project was starting in 2014 and the first milestone reached was the elaboration of the consistent fuel cycle architecture described above and the delineation of the R\&D programme. The most urgent near-term activity in parallel to the detailed implementation of the programme actions is to consolidate the requirements and to compile quantitative input numbers, in particular with regard to the input streams coming from the tritium extraction system and the coolant loops of the breeding blankets (for the different breeding concepts) as well as with regard to the total machine throughput (including PEGs).

For the DEMO tritium systems, a pre-conceptual integrated plant design activity supported by one or two focal experimental activities is envisaged to run until 2018. In the sub-project matter integration, emphasis will be laid on theoretical development and output from existing experimental devices. A case will be made whether or not a European pellet injector test bed is needed to advance the systems in view of DEMO with lower risk. For the vacuum systems, the new concepts for liquid ring pumps and diffusion pumps for DEMO are foreseen to be tested under tritium conditions (at JET in 2017 [57] and at the Tritium Laboratory Karlsruhe at 2018, respectively) so that a very high technical readiness level can be demonstrated.

\section{Acknowledgments}

This work has been carried out within the framework of the EUROfusion Consortium and has received funding from the EURATOM research and training programme 2014-2018 under grant agreement No. 633053. The views and opinions expressed herein do not necessarily reflect those of the European Commission.

The authors would like to acknowledge the contributions made to the generation of the material for this paper by the following staff at CCFE, ENEA and KIT: Volker Hauer, Bernhard Plöckl, Sebastian RussellSmith, Alessia Santucci, Rebecca Shaw and also those in these organisations who have taken part in supporting group activities during ranking exercises.

\section{References}

[1] F. Romanelli et al., A roadmap to the realisation of fusion energy, EFDA Garching, Germany, 2012.

[2] D. Babineau et al., Review of the ITER fuel cycle, IAEA Fusion Energy Conf., Daejon, Korea, Oct. 2010, available online: http://wwwpub.iaea.org/mtcd/meetings/PDFplus/2010/cn180/c n180_papers/itr_2-2.pdf

[3] I. Ricapito, P. Calderoni, Y. Poitevin. A. Aiello, FED 89 (2014) 1469-1475.

[4] G. Federici et al., Overview of the design approach and prioritization of R\&D activities towards an EU DEMO, This Conference.

[5] G. Federici et al., FED 89 (2014) 882-889.

[6] R. Wenninger et al., DEMO exhaust challenges beyond ITER, Proc. of the $42^{\text {nd }}$ EPS Conference, Lisbon, Portugal, 2015.

[7] Chr. Day, Th. Giegerich, FED 88 (2013) 616-620.

[8] P. Pearce, S. Friedenthal, A practical approach for modelling submarine subsystem architecture in SysML, Proc. $2^{\text {nd }}$ SIA Science, Technology and Engineering Conference, Adelaide, Australia, Oct. 2013, pp. 347-360.

[9] T. Pinna, F. Borgognoni, Fusion Sci. Technol. 67 (2015) 531-534.

[10] D. vanHoutte, F. Sagot, K. Okayama, K. Blackler, FED 87 (2012) 652-656.

[11] W. Biel et al., DEMO diagnostics and burn control, FED (2015), in press.

[12] M. Glugla et al., FED 82 (2007) 472-487.

[13] C.L. Dym, W.H. Wood, M.J. Scott, Research in Engineering Design 13 (2002) 236-242.

[14] Design engineering methodics, VDI (German Engineering Society) Guideline 2225-3, Beuth, Berlin, 1998.

[15] F. Toci et al., FED 28 (1995) 373-377.

[16] J.D. Baker, D.H. Meikrantz, R.J. Pawelko, R.A. Anderl, D.G. Tuggle, J. Vac. Sci. Technol. A 12 (1994) 548.

[17] B. Bornschein et al., FED 75-79 (2005) 645-650.

[18] J.L. Anderson, K. Okuno, FED 28 (1995) 183-189.

[19] S. Welte, D. Demange, R. Wagner, FED 85 
(2010)1320-1325.

[20] S. Tosti et al., FED 86 (2011) 2180-2183.

[21] D. Demange et al., Catalysis Today 156 (2010) 140145.

[22] O. Borisevich, D. Demange, M. Kind, X. Lefebvre, Fusion Science Technol. 67 (2015) 262-265.

[23] I. Ricapito et al., Fusion Sci. Technol. 67 (2015) 543-546

[24] I. Cristescu et al., FED 89 (2014) 1524-1528.

[25] A.N. Ezoubtchenko, , H. Akazuka, M. Suzuki, Progress in Nucl. Energy 32 (1998) 729-735.

[26] G. Vasaru, Tritium isotope separation, CRC Press, 1993.

[27] H. Tanaka, H. Kanoh, M. Yudasaka, S. Iijima, K. Kaneko, J. Am. Chem. Soc. 127 (2005) 7511-7516.

[28] L.K. Heung, Demonstration of the next generation TCAP Hydrogen isotope separation process, report SRNL-STI-2009-22, SRNL, US, 2009.

[29] D. Ducret et al., FED 58-59 (2001) 417-421.

[30] N. Hanada, T. Ichikawa, S. Hino, H. Fuji, J. Alloys Compounds 420 (2006) 46-49.

[31] S.H. Yun et al., J. Plasma Fus. Res. 10 (2013) 54-63.

[32] D. Demange et al., FED 89 (2014) 1219-1222.

[33] A. Ciampichetti et al., FED 85 (2010) 2033-2039.

[34] A.N. Perevezentsev et al., FED 61-62 (2002) 585589.

[35] A.N Perevezentsev et al., FED 85 (2010) 12061210.

[36] S.K. Combs et al., FED 87 (2012) 634-640.

[37] P.B. Parks, F.W. Perkins, Nucl. Fusion 46 (2006) 770-780.

[38] X. Wang et al., Laser and Particle Beams 27 (2009) 629-634.

[39] R. Raman, FED 83 (2008) 1368-1374.

[40] W. Liu, S.C. Hsu, Nucl. Fusion 51 (2010) 073026.

[41] E. Belonohy et al., Nucl. Fusion 48 (2008) 065009.

[42] P.T. Lang et al., Considerations on the pellet DEMO fuelling system, FED (2015), in press.

[43] A. Geraud et al. FED 88 (2013) 1064-1068.

[44] A. Frattolillio et al., Rev. Sci. Instr. 67 (1996) 18341841.

[45] B. Ploeckl et al., The enhanced pellet centrifuge launcher at ASDEX Upgrade: Advanced operation and application as technology test facility for ITER and DEMO, FED (2015), in press.

[46] International Standard ISO 3529-1:1981, Vacuum Technology - General Terms.

[47] Chr. Day, Th. Giegerich, IEEE Trans. Plasma Science 42 (2014) 1058-1071.

[48] A. Mack, D. Perinic, FED 28 (1995) 319-323.

[49] M. Kovari, R. Clarke, T. Shephard, FED 88 (2013) 3293-3298.

[50] A.I. Livshits, M.E. Notkin and A.A. Samartsev, J. Nucl. Mater. 170 (1990) 79-94.

[51] C.L. Volkers, V.P. Gede, Transfer operations with tritium - a review, Proc. 23rd Conf. on Remote Systems Technology, San Francisco, CA, USA, 1975.

[52] M. Lind, F. Steinhaus, Development of a large linear jet mercury diffusion pump, Scientific report MTA14, Livermore Research Labaratory, Livermore, CA, USA, 1953.

[53] C.J. Caldwell-Nichols, M. Glugla, S. Welte, D.K.
Murdoch, FED 75-79 (2005) 663-666.

[54] T. Giegerich, Chr. Day, FED 89 (2014) 1476-1481.

[55] Chr. Day et al., FED 89 (2014) 1505-1509.

[56] Chr. Day, S. Varoutis, Y. Igitkhanov, Effect of the dome on the collisional neutral gas flow in the DEMO divertor, accepted for publication in Trans. Plasma Science (2015).

[57] T. Giegerich et al., Advanced design of the Mechanical Tritium Pumping System for JET DTE2, This Conference. 